\title{
Adaptive Randomness: A New Population Initialization Method
}

\author{
Weifeng Pan, ${ }^{1}$ Kangshun Li, ${ }^{2}$ Muchou Wang, ${ }^{3}$ Jing Wang, $^{4}$ and Bo Jiang ${ }^{1}$ \\ ${ }^{1}$ School of Computer Science and Information Engineering, Zhejiang Gongshang University, Hangzhou, Zhejiang 310018, China \\ ${ }^{2}$ College of Information, South China Agricultural University, Guangzhou, Guangdong 510642, China \\ ${ }^{3}$ Wenzhou University Library, Wenzhou University, Wenzhou, Zhejiang 325035, China \\ ${ }^{4}$ School of Software and Communication Engineering, Jiangxi University of Finance and Economics, Nanchang, Jiangxi 330013, China
}

Correspondence should be addressed to Weifeng Pan; panweifeng1982@gmail.com

Received 2 August 2014; Accepted 15 October 2014; Published 13 November 2014

Academic Editor: Erik Cuevas

Copyright (C) 2014 Weifeng Pan et al. This is an open access article distributed under the Creative Commons Attribution License, which permits unrestricted use, distribution, and reproduction in any medium, provided the original work is properly cited.

Population initialization is a crucial task in population-based optimization methods, which can affect the convergence speed and also the quality of the final solutions. Generally, if no a priori information about the solutions is available, the initial population is often selected randomly using random numbers. This paper presents a new initialization method by applying the concept of adaptive randomness (AR) to distribute the individuals as spaced out as possible over the search space. To verify the performance of AR, a comprehensive set of 34 benchmark functions with a wide range of dimensions is utilized. Conducted experiments demonstrate that AR-based population initialization performs better than other population initialization methods such as random population initialization, opposition-based population initialization, and generalized opposition-based population initialization in the convergence speed and the quality of the final solutions. Further, the influences of the problem dimensionality, the new control parameter, and the number of trial individuals are also investigated.

\section{Introduction}

Evolutionary algorithms (EAs) are population-based stochastic optimization algorithms. For each optimization problem, they maintain a set of candidate solutions to play the role of individuals in a population, perform crossover and mutation operations on this set to generate different solutions, and use a fitness function to determine the environment within which the solutions live. In the last decade, EAs have been applied successfully to solve many real-world and benchmark optimization problems. However, as populationbased algorithms, EAs such as the genetic algorithm (GA) [1] and differential evolution (DE) [2,3] all have common drawbacks-long computational time and premature convergence, especially when the solution space is hard to explore.

Since reducing computation time needed to reach optimal solutions and improve the quality of the final solutions would be beneficial, many efforts have been already done. However, most of the work mainly focused on the introduction and improvement of selection mechanisms, crossover and mutation operators, parameter adjustments, and some other hybrid strategies. If no information about the solution is available, the most commonly used method to generate the initial population is random initialization. Little work has been done on the population initialization, even though it is a crucial task in EAs and can affect the convergence speed and also the quality of the final solution. Maaranen et al. [4] used quasirandom sequences to generate the initial population for GAs. The experimental results showed that their approach could improve the quality of the final solutions but with no noteworthy improvement for convergence speed. Rahnamayan et al. [5] proposed an opposition-based population initialization method, which achieved a fast convergence speed. Wang et al. [6] presented a population initialization method based on space transformation search (in their following work, such a method is renamed as generalized opposition-based initialization [7]). Experimental results showed that their approach when combined with other strategies outperformed the traditional random initialization and opposition-based initialization.

This paper proposes a new approach for population initialization by employing the adaptive randomness (AR) to 
improve the quality of the final solutions and also accelerate the convergence speed. AR initialization is an enhanced version of random initialization. It is simple and easy to be implemented. The main idea of $\mathrm{AR}$ is to make use of the difference between individuals to make them more evenly spread over the entire search space and then a better approximation for the current candidate solution is obtained. Although this paper only embeds the AR for population initialization of classical DE, the idea is general enough to be applied to all other EAs. Experimental results on 34 well-known benchmark problems show that the proposed approach performs better than the random initialization, opposition-based initialization, and generalized oppositionbased initialization both in the quality of the final solutions and the convergence speed.

The remainder of this paper is organized as follows: in Section 2, the concept of AR is briefly explained. In Section 3, the classical DE is briefly reviewed. In Section 4, the proposed AR-based population initialization algorithm is presented. Experimental results are given in Section 5 with focus on the test functions used, parameter setting, results, and results' analysis. In Section 6, we conclude the work and all benchmark functions are listed in the appendix.

\section{Adaptive Randomness}

Traditionally, EAs imitate natural evolution in a population. The population is a set of candidate solutions to an optimization problem, making us consider several solutions at the same time. The population evolves from one generation to another as the individuals are crossbred and mutated until the predefined criteria are satisfied. If no a priori information about the solution is available, the initial population is often selected randomly using random numbers [4]. Obviously, the computation time is directly related to the distance of the random numbers from optimal solutions [5].

In practice, random numbers cannot be generated algorithmically. The algorithmically generated numbers (usually called pseudorandom numbers) only try to imitate random numbers. However, it is usually more important that the numbers are as evenly distributed as possible than that they imitate random numbers [4], for they provide much more information about the fitness function. This forms the basis of our approach for population initialization, namely, adaptive randomness (AR).

AR slightly modifies the random initialization by controlling the individuals that can come into the initial population. When adding a new individual to the initial population, AR needs to make sure that the individual should not be too close to any of the previous individuals already in the initial population.

To achieve this, AR should maintain two sets of individuals, that is, partial initial population (PP) and set of trial individuals (ST). Before concentrating on AR-based population initialization, we define the two sets first.

Definition 1. Let $P(\mathrm{ps})=\left\{X_{1}, X_{2}, \ldots, X_{\mathrm{ps}}\right\}$ be the initial population of a specific optimization method, where $p s$ is the population size and $X_{i}(i=1,2, \ldots, \mathrm{ps})$ is the candidate solution in a $D$-dimensional space. Then PP is defined by $\mathrm{PP} \subseteq P$.

Definition 2. ST $(k)=\left\{Y_{1}, Y_{2}, \ldots, Y_{k}\right\}$ is the set of $k$ trial individuals such that $\mathrm{ST} \cap \mathrm{PP}=\emptyset$. Each trial individual $Y_{i}(i=1,2, \ldots, k)$ is randomly chosen from the $D$-dimensional search space and $k$ is the predefined number of trial individuals.

Obviously trial individuals are those individuals that are randomly generated from the search space but have not been added into PP.

To distribute the individuals in $\mathrm{PP}$ as spaced out as possible, when adding a new individual into $\mathrm{PP}, \mathrm{AR}$ first generates $k$ trial individuals to form ST and then the trial individual that is farthest away from all individuals in PP is selected to be added into PP. Such a process is repeated until the number of individuals in PP reaches ps.

In $\mathrm{AR}$ the distance between every pair of individuals $X_{i}\left(x_{i 1}, x_{i 2}, \ldots, x_{i D}\right) \in \mathrm{PP}$ and $Y_{j}\left(y_{j 1}, y_{j 2}, \ldots, y_{j D}\right) \in \mathrm{ST}$ is calculated by Euclidean distance as

$$
d\left(X_{i}, Y_{j}\right)=\left\|X_{i}, Y_{j}\right\|=\sqrt{\sum_{t=1}^{D}\left(x_{i t}-y_{j t}\right)^{2}} .
$$

So when adding a new individual into PP the trial individual $Y_{n} \in$ ST will be chosen such that for all $j \in$ $\{1,2, \ldots, k\}$,

$$
\min _{1 \leq i \leq|\mathrm{PP}|} d\left(X_{i}, Y_{n}\right) \geq \min _{1 \leq i \leq|\mathrm{PP}|} d\left(X_{i}, Y_{j}\right),
$$

where $|\mathrm{PP}|$ counts the number of individuals in $\mathrm{PP}$ and min returns the minimum value in a set of values. The rationale for using (2) is to evenly distribute the individuals in initial population by maximizing the minimum distance between the trial individual selected and the individuals already in PP.

Figure 1 gives a simple example to show you how to select the trial individual to update PP. Assume that PP already has two individuals (i.e., $X_{1}$ and $X_{2}$ ) and we generate ST with two (suppose that $k=2$ ) trial individuals (i.e., $Y_{1}$ and $\left.Y_{2}\right)$. According to (1), we can obtain $d\left(X_{1}, Y_{1}\right)=7.076722$, $d\left(X_{2}, Y_{1}\right)=4.060788, d\left(X_{1}, Y_{2}\right)=5.507268$, and $d\left(X_{2}, Y_{2}\right)=$ 3.2. So $\min \left(\left\{d\left(X_{1}, Y_{1}\right), d\left(X_{2}, Y_{1}\right)\right\}\right)=d\left(X_{2}, Y_{1}\right)=4.060788$ and $\min \left(\left\{d\left(X_{1}, Y_{2}\right), d\left(X_{2}, Y_{2}\right)\right\}\right)=d\left(x_{2}, Y_{2}\right)=3.2$. Since $d\left(X_{2}, Y_{1}\right)>d\left(X_{2}, Y_{2}\right)$, the trial individual $Y_{1}$ will be added into PP.

Before introducing the AR-based population initialization algorithm, the classical DE is briefly reviewed in the following section.

\section{Brief Review of the Classical DE}

DE, firstly proposed by Storn and Price in [2, 3], is a population-based stochastic optimization algorithm and has been successfully used in both benchmark test functions and real-world applications. It is simple yet effective and robust. A plethora of experimental studies show its better performance than other EAs.

The proposed algorithm is also based on this DE scheme. Let us assume that $X_{i}(t)(i=1,2, \ldots, \mathrm{ps})$ is the $i$ th individual 


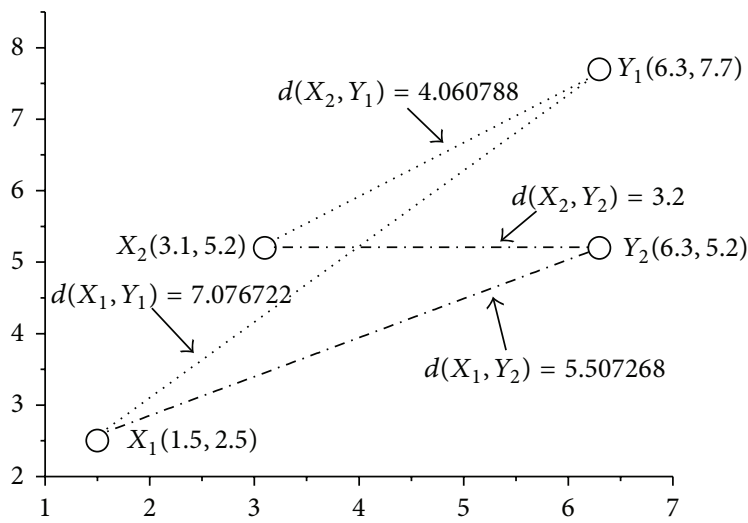

FIgURE 1: Illustration of the selection of a trial individual into PP in a two-dimensional space.

in population $P(t)$, where ps is the population size, $t$ is the generation index, and $P(t)$ is the population in the $i$ th generation. The main idea of $\mathrm{DE}$ is to generate trial vectors. Mutation and crossover are used to produce new trial vectors, and selection determines which of the vectors will be successfully selected into the next generation.

For classical DE (DE/rand/1/bin), the mutation, crossover, and selection operators can be defined as follows.

Mutation. For each vector $X_{i}(t)$ in generation $t$, a mutant vector $V_{i}(t+1)$ is defined by

$$
\begin{array}{r}
V_{i}(t+1)=X_{i 1}(t)+F\left(X_{i 2}(t)-X_{i 3}(t)\right) \\
i \neq i_{1} \neq i_{2} \neq i_{3},
\end{array}
$$

where $i=1,2, \ldots$, ps and $i_{1}, i_{2}$, and $i_{3}$ are randomly selected integer indices from [1,ps]. Further, $i \neq i_{1} \neq i_{2} \neq i_{3}$, so the population size ps satisfies ps $\geq 4$. $F \in[0,2]$ is a real number which determines the amplification of the differential variation $\left(X_{i 2}(t)-X_{i 3}(t)\right)$. Larger values of $F$ result in higher diversity in the generated population and lower values in faster convergence.

Crossover. As many other EAs do, DE also takes a crossover operation to increase the diversity of population and generate the trial vectors. The trial vector can be defined as

$$
U_{i}(t+1)=\left(U_{i 1}(t+1), U_{i 2}(t+1), \ldots, U_{i D}(t+1)\right),
$$

where $D$ is the problem dimension. The classical DE uses the $\mathrm{DE} / \mathrm{rand} / 1 /$ bin scheme to generate the trial vector

$$
U_{i j}(t+1)= \begin{cases}V_{i j}(t+1), & \text { if } \operatorname{rand}_{j}(0,1) \leq \mathrm{CR} \\ X_{i j}(t), & \text { otherwise, }\end{cases}
$$

where CR $\in[0,1]$ is the predefined crossover probability, $\operatorname{rand}_{j}(0,1)$ is a random number within $[0,1]$ for the $j$ th dimension, and $j \in\{1,2, \ldots, D\}$ is a random parameter index.

Selection. A greedy selection mechanism is used as

$$
X_{i}(t+1)= \begin{cases}U_{i}(t+1), & \text { if } f\left(U_{i}(t+1)\right) \leq f\left(X_{i}(t)\right) \\ X_{i}(t), & \text { otherwise, }\end{cases}
$$

where $f(\cdot)$ is the fitness function. Without loss of generality, this paper only considers minimization problems. If, and only if, the trial vector $U_{i}(t+1)$ is better than $X_{i}(t)$ (i.e., $f\left(U_{i}(t+\right.$ $\left.1)) \leq f\left(X_{i}(t)\right)\right), X_{i}(t+1)$ is set to $U_{i}(t+1)$; otherwise, the $X_{i}(t+$ 1) remains unchanged; that is, $X_{i}(t+1)=X_{i}(t)$. Hence the population either gets better or remains the same with respect to the fitness function but never deteriorates.

Though there are many variants of DE [2,3], to maintain a general comparison, this paper only uses the classical $\mathrm{DE}$ in the conducted experiments to demonstrate the improvement of the convergence speed and the quality of the final solutions by using AR-based population initialization.

\section{The Proposed AR-Based Population Initialization Algorithm}

For a specific optimization problem, when lacking a priori information about the solutions, the initial population is usually created using random numbers. AR makes full use of the distance information during the process of population initialization. By applying the AR strategy, we can distribute the individuals as spaced out as possible and obtain a better approximation for the current candidate solutions. So instead of using a pure random initialization, we propose the following AR-based population initialization algorithm (see Algorithm 1).

As is shown in Algorithm 1, PP is initially empty and the first individual of PP is randomly chosen from the search space. During population initialization, PP will be incrementally updated with the individuals selected from ST until the number of individuals in PP reaches the population size ps.

The flowcharts of $\mathrm{DE}$ with random population initialization, opposition-based population initialization, generalized opposition-based population initialization, and AR-based population initialization are shown in Figure 2.

AR-based population initialization will be embedded in the classical DE in Section 5 to show its effectiveness in the improvement of the convergence speed and the quality of the final solutions.

\section{Empirical Study}

To investigate the effectiveness of the proposed AR-based population initialization algorithm in the improvement of convergence speed and the final solution, we embedded it in the classical DE and conducted controlled experiments. Our experiments were carried out on a PC at $2.3 \mathrm{GHz}$ with $2 \mathrm{~GB}$ of RAM.

In the following subsections, we provide details on the test functions of our study (Section 5.1), parameter settings 


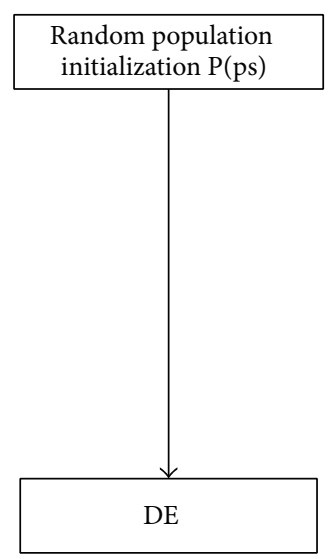

(a)

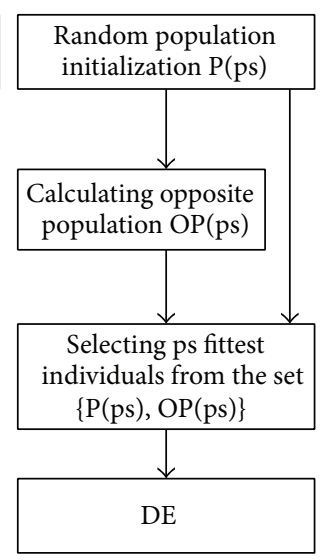

(b)

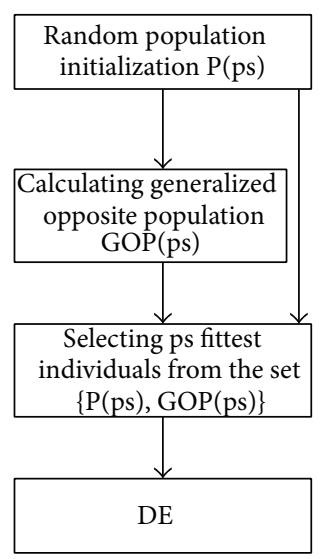

(c)

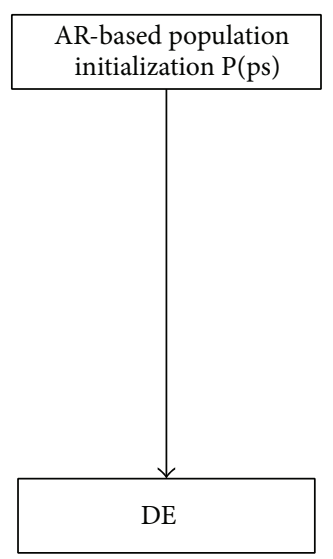

(d)

FIGURE 2: DE with (a) random population initialization, (b) opposition-based population initialization, (c) generalized opposition-based population initialization, and (d) AR-based population initialization.

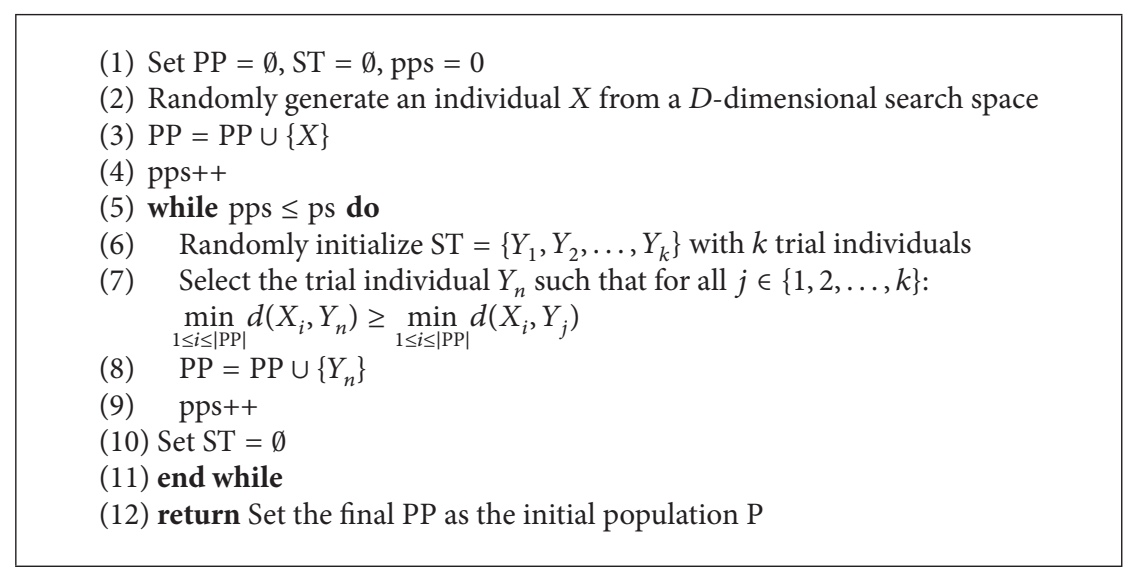

Algorithm 1: AR-based population initialization algorithm.

(Section 5.2), and our experiment results and analysis (Section 5.3).

5.1. Test Functions. In order to compare the convergence speed and the quality of the final solutions of DE with random population initialization $\left(\mathrm{DE}_{\mathrm{r}}\right), \mathrm{DE}$ with oppositionbased population initialization $\left(\mathrm{DE}_{\mathrm{o}}\right), \mathrm{DE}$ with generalized opposition-based population initialization $\left(\mathrm{DE}_{\mathrm{go}}\right)$, and $\mathrm{DE}$ with AR-based population initialization $\left(\mathrm{DE}_{\mathrm{ar}}\right)$, a comprehensive test set with 34 numerical benchmark functions is employed. The test set includes well-known unimodal as well as highly multimodal minimization problems $[8,9]$. The definition, the range of search space, and also the global optimum(s) for each function are given in the appendix. The dimensionality of these problems varies from 2 to 100 , covering a wide range of problem complexity.

5.2. Parameter Settings. For all the conducted experiments, the parameters of the classical DE, namely, population size (ps), differential amplification factor $(F)$, crossover probability constant (CR), and maximum number of function calls $\left(\mathrm{MAX}_{\mathrm{NFC}}\right)$, if no a change is mentioned, are fixed to 100 , $0.5,0.9$, and $10^{6}$, respectively. Such a setting follows the suggestions given in the literature (e.g., [10-12]). And the parameter $k$ of ST in AR-based population initialization is set to 3 unless a change is mentioned.

5.3. Results and Analysis. The experiments are categorized as follows. In Section 5.3.1, $\mathrm{DE}_{\mathrm{r}}, \mathrm{DE}_{\mathrm{o}}, \mathrm{DE}_{\mathrm{go}}$, and $\mathrm{DE}_{\mathrm{ar}}$ are compared in terms of convergence speed and robustness. In Section 5.3.2, $\mathrm{DE}_{\mathrm{r}}, \mathrm{DE}_{\mathrm{o}}, \mathrm{DE}_{\mathrm{go}}$, and $\mathrm{DE}_{\mathrm{ar}}$ are compared in terms of the quality of the final solution. In Section 5.3.3, the effect of problem dimensionality is investigated. In Section 5.3.4, the effect of parameter $k$ is studied. All the experiments are conducted 1,000 times with different random seeds, and the average results throughout the optimization runs are recorded. It should be noted that, in the experiments, we find that for a small value of conducted times, the values of evaluation times and the final solutions are not stable. 
5.3.1. Comparison of $D E_{\mathrm{r}}, D E_{\mathrm{o}}, D E_{\mathrm{go}}$, and $D E_{\mathrm{ar}}$ in Terms of Convergence Speed and Robustness. By the suggestions in the literature (e.g., $[5,6,9])$, we compare the convergence speed of $\mathrm{DE}_{\mathrm{r}}, \mathrm{DE}_{\mathrm{o}}, \mathrm{DE}_{\mathrm{go}}$, and $\mathrm{DE}_{\mathrm{ar}}$ by measuring the number of function calls (NFC). For each optimization problem, NFC is recorded when a specific algorithm reduces the best value to a value smaller than the value-to-reach (VTR) before meeting $\mathrm{MAX}_{\mathrm{NFC}}$. In order to minimize the effect of the stochastic nature of the algorithms, all the reported NFCs are averaged over 1,000 independent trials. Obviously, a smaller NFC means a higher convergence speed. In order to compare the convergence speed between two specific algorithms, we introduce another metric, acceleration rate (ARE), which is defined as

$$
\mathrm{ARE}=\frac{\mathrm{NFC}_{\mathrm{algA}}}{\mathrm{NFC}_{\mathrm{algB}}},
$$

where $\mathrm{NFC}_{\mathrm{algA}}$ and $\mathrm{NFC}_{\mathrm{algB}}$ are the NFCs for the two algorithms algA and algB (algA and algB are all chosen from $\left.\left\{\mathrm{DE}_{\mathrm{r}}, \mathrm{DE}_{\mathrm{o}}, \mathrm{DE}_{\mathrm{go}}, \mathrm{DE}_{\mathrm{ar}}\right\}\right)$. So $\mathrm{ARE}>1$ means that algB is faster. The VTR is set to $10^{-6}$ for all benchmark functions. The same setting has been used in the literature (e.g., $[13,14])$.

We also compare the robustness of $\mathrm{DE}_{\mathrm{r}}, \mathrm{DE}_{\mathrm{o}}, \mathrm{DE}_{\mathrm{go}}$, and $\mathrm{DE}_{\mathrm{ar}}$ by measuring the success rate (SR) [13]. In the current work, a successful running means that a specific algorithm successfully reaches the VTR for each test function in the allowed $\mathrm{MAX}_{\mathrm{NFC}}$. So SR can be calculated as

$$
\mathrm{SR}=\frac{\text { number of times reached VTR }}{\text { total number of trials }} .
$$

SR is a commonly used metric to characterize the robustness of a specific algorithm; that is, a larger SR means that the algorithm is more robust.

Further the average NFC $\left(\mathrm{NFC}_{\text {avg }}\right)$, the average SR $\left(\mathrm{SR}_{\mathrm{avg}}\right)$, and the average $\mathrm{ARE}\left(\mathrm{ARE}_{\mathrm{avg}}\right)$ over the $n$ test functions are calculated as

$$
\begin{aligned}
\mathrm{NFC}_{\mathrm{avg}} & =\frac{1}{n} \sum_{i=1}^{n} \mathrm{NFC}_{i}, \\
\mathrm{ARE}_{\mathrm{avg}} & =\frac{1}{n} \sum_{i=1}^{n} \mathrm{ARE}_{i}, \\
\mathrm{SR}_{\mathrm{avg}} & =\frac{1}{n} \sum_{i=1}^{n} \mathrm{SR}_{i} .
\end{aligned}
$$

Table 1 summarizes the numerical results when solving the 34 benchmark functions shown in the appendix. The best result of NFC for each function is highlighted in boldface and $\mathrm{NFC}_{\text {avg }}, \mathrm{SR}_{\mathrm{avg}}$, and $\mathrm{ARE}_{\text {avg }}$ are shown in the last row. Since comparing the algorithms with different SR values seems meaningless, the reported average values are calculated only on the functions where all the algorithms have the same success rate.

As seen, $\mathrm{DE}_{\mathrm{ar}}$ outperforms $\mathrm{DE}_{\mathrm{r}}$ on 22 test functions (about $64.7 \%$ of the problems). Though $\mathrm{DE}_{\mathrm{r}}$ is faster than $\mathrm{DE}_{\mathrm{ar}}$ on 4 functions (i.e., $f_{6}, f_{16}, f_{26}$, and $f_{31}$ ), its SR is worse.
Except for $f_{6}$, the rest 3 functions are functions with a low dimensionality $(D \leq 10)$. $\mathrm{DE}_{\mathrm{ar}}$ outperforms $\mathrm{DE}_{\mathrm{o}}$ on 22 test functions (about $64.7 \%$ of the problems), while $\mathrm{DE}_{\mathrm{o}}$ surpasses $\mathrm{DE}_{\mathrm{ar}}$ only on 4 functions (i.e., $f_{6}, f_{18}, f_{26}$, and $f_{29}$ ). Though $\mathrm{DE}_{\mathrm{o}}$ is faster than $\mathrm{DE}_{\mathrm{ar}}$ on $f_{6}$ and $f_{26}$, its SR is worse. $\mathrm{DE}$ ar outperforms $\mathrm{DE}_{\mathrm{go}}$ on 24 test functions (about $70.6 \%$ of the problems), while $\mathrm{DE}_{\mathrm{go}}$ surpasses $\mathrm{DE}_{\mathrm{ar}}$ only on $f_{4}$ and $f_{6}$. Though $\mathrm{DE}_{\mathrm{go}}$ is faster than $\mathrm{DE}_{\mathrm{ar}}$ on $f_{6}$, its SR is worse. All the algorithms fail to solve 6 functions (i.e., $f_{19}, f_{21}, f_{22}, f_{23}$, $f_{24}$, and $\left.f_{25}\right)$. On $f_{15}$ and $f_{30}$, only $\mathrm{DE}_{\text {ar }}$ can solve them with a very small ARE while the other three algorithms all fail. The $\mathrm{ARE}_{\mathrm{avg}}$ between $\mathrm{DE}_{\mathrm{r}}$ and $\mathrm{DE}_{\mathrm{ar}}$ is 1.0116, which means that $\mathrm{DE}_{\mathrm{ar}}$ is on average $1.16 \%$ faster than $\mathrm{DE}_{\mathrm{r}}$. Similarly, $\mathrm{DE}_{\mathrm{ar}}$ is on average $1.52 \%$ faster than $\mathrm{DE}_{\mathrm{r}}$ and $\mathrm{DE}_{\mathrm{ar}}$ is on average $1.38 \%$ faster than $\mathrm{DE}_{\mathrm{go}}$.

So we can conclude that $\mathrm{DE}_{\mathrm{ar}}$ shows better convergence speed than the other 3 algorithms with the same parameter settings and fixing the $k$ for the $\mathrm{DE}_{\mathrm{ar}}(k=3)$. Some sample bar charts for the performance comparison of the 4 algorithms are given in Figure 3.

5.3.2. Comparison of $D E_{\mathrm{r}}, D E_{\mathrm{o}}, D E_{\mathrm{go}}$, and $D E_{\mathrm{ar}}$ in Terms of the Quality of Final Solutions. In this section, $\mathrm{DE}_{\mathrm{ar}}$ is compared with $\mathrm{DE}_{\mathrm{r}}, \mathrm{DE}_{\mathrm{o}}$, and $\mathrm{DE}_{\mathrm{go}}$ with respect to the quality of the final solutions. All the experiments were conducted 1,000 times, and the mean function error value and standard deviation of the results are recorded. The results of the $4 \mathrm{DE}$ algorithms on the 34 test problems are presented in Tables 2 and 3, where "Mean" indicates the mean function error value and "Std. Dev." stands for the standard deviation. The best results among the $4 \mathrm{DE}$ algorithms are shown in boldface.

From the results, it can be seen that $\mathrm{DE}_{\mathrm{ar}}$ achieves better results than $\mathrm{DE}_{\mathrm{r}}, \mathrm{DE}_{\mathrm{o}}$, and $\mathrm{DE}_{\mathrm{go}}$ on 24 test functions (about $70.5 \%$ of the test functions). $\mathrm{DE}_{\mathrm{ar}}$ achieves the same performance as the $\mathrm{DE}_{\mathrm{go}}$ on function $f_{26}$, and they are both much better than the other algorithms on this problem. For the rest of the 9 functions, all the algorithms achieve the same results.

To compare the performance of multiple algorithms on the test suite, the average ranking of the Friedman test is conducted by the suggestions considered in $[7,15]$. Table 4 shows the average ranking of the $4 \mathrm{DE}$ algorithms on functions $f_{1}-f_{34}$. These algorithms can be sorted by average ranking into the following order: $\mathrm{DE}_{\mathrm{ar}}, \mathrm{DE}_{\mathrm{r}}, \mathrm{DE}_{\mathrm{go}}$, and $\mathrm{DE}_{\mathrm{o}}$. It means that $\mathrm{DE}_{\mathrm{ar}}$ and $\mathrm{DE}_{\mathrm{o}}$ are the best and worst ones among the four algorithms, respectively. So as seen, although opposition-based population initialization can accelerate the convergence speed on some test problems, when compared with $\mathrm{DE}_{\mathrm{r}}$, it cannot improve the quality of the final solutions. So if we want to obtain a high quality solution, oppositionbased population initialization cannot be used alone.

To investigate the significant differences between the behavior of two algorithms, we conduct four tests, that is, Nemenyi's, Holm's, Shaffer's, and Bergmann-Hommel's [7, 15]. For each test, we calculate the adjusted $P$ values on pairwise comparisons of all algorithms. Table 5 shows the results of adjusted $P$ values. Under the null hypothesis, the two algorithms are equivalent. If the null hypothesis is rejected, then the performances of these two algorithms are 


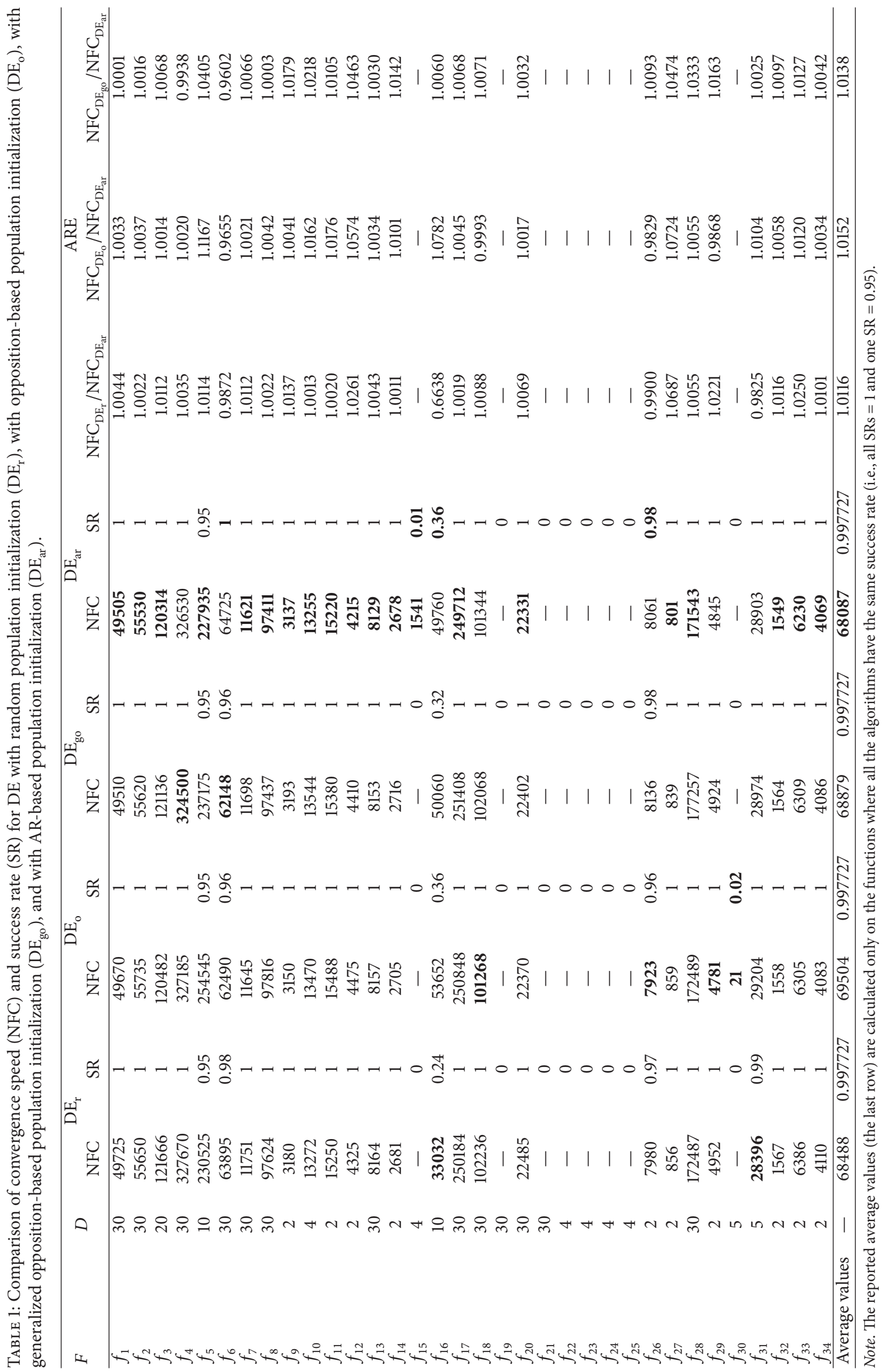


TABLE 2: Comparison among the $4 \mathrm{DE}$ algorithms on test problems $f_{1}-f_{22}$, where "Mean" indicates the mean function error value and "Std. Dev." stands for the standard deviation. The best results among the four algorithms are shown in boldface.

\begin{tabular}{|c|c|c|c|c|c|c|}
\hline & $D$ & & $\mathrm{DE}_{\mathrm{r}}$ & $\mathrm{DE}_{\mathrm{o}}$ & $\mathrm{DE}_{\mathrm{go}}$ & $\mathrm{DE}_{\mathrm{ar}}$ \\
\hline \multirow{2}{*}{$f_{1}$} & \multirow{2}{*}{30} & Mean & $8.81228 e-009$ & $8.81301 e-009$ & $8.79674 e-009$ & $8.77417 e-009$ \\
\hline & & Std. Dev. & $1.10323 e-009$ & $8.84418 e-010$ & $1.08043 e-009$ & $8.7124 e-010$ \\
\hline \multirow{2}{*}{$f_{2}$} & \multirow{2}{*}{30} & Mean & $9.04527 e-009$ & $9.10281 e-009$ & $9.06893 e-009$ & $8.92437 e-009$ \\
\hline & & Std. Dev. & $7.96893 e-010$ & $6.70026 e-010$ & $7.70684 e-010$ & $7.79631 e-010$ \\
\hline \multirow{2}{*}{$f_{3}$} & \multirow{2}{*}{20} & Mean & $9.34818 e-009$ & $9.39706 e-009$ & $9.38949 e-009$ & $9.33293 e-009$ \\
\hline & & Std. Dev. & $5.33216 e-010$ & $4.83679 e-010$ & $5.58047 e-010$ & $5.48986 e-010$ \\
\hline \multirow{2}{*}{$f_{4}$} & \multirow{2}{*}{30} & Mean & $9.26684 e-009$ & $9.32035 e-009$ & $9.3298 e-009$ & $9.11962 e-009$ \\
\hline & & Std. Dev. & $7.17271 e-010$ & $6.50894 e-010$ & $5.82968 e-010$ & $7.65305 e-010$ \\
\hline \multirow{2}{*}{$f_{5}$} & \multirow{2}{*}{10} & Mean & 0.049748 & 0.049748 & 0.049748 & 0.049748 \\
\hline & & Std. Dev. & 0.22248 & 0.22248 & 0.22248 & 0.22248 \\
\hline \multirow{2}{*}{$f_{6}$} & \multirow{2}{*}{30} & Mean & 0.000172542 & 0.00029585 & 0.000320462 & $8.80458 e-009$ \\
\hline & & Std. Dev. & 0.00122635 & 0.00146403 & 0.00159228 & $1.20596 e-009$ \\
\hline \multirow{2}{*}{$f_{7}$} & \multirow{2}{*}{30} & Mean & $6.24585 e-009$ & $6.62518 e-009$ & $6.86516 e-009$ & $6.20522 \mathrm{e}-009$ \\
\hline & & Std. Dev. & $2.32947 e-009$ & $2.28414 e-009$ & $2.42006 e-009$ & $2.35472 e-009$ \\
\hline \multirow{2}{*}{$f_{8}$} & \multirow{2}{*}{30} & Mean & $9.3102 e-009$ & $9.33032 e-009$ & $9.2814 e-009$ & $9.25572 \mathrm{e}-009$ \\
\hline & & Std. Dev. & $4.90595 e-010$ & $5.23764 e-010$ & $6.79597 e-010$ & $5.84208 e-010$ \\
\hline \multirow{2}{*}{$f_{9}$} & \multirow{2}{*}{2} & Mean & $4.46327 e-009$ & $4.39462 e-009$ & $4.67599 e-009$ & $4.31941 \mathrm{e}-009$ \\
\hline & & Std. Dev. & $2.95843 e-009$ & $3.02071 e-009$ & $2.72012 e-009$ & $2.98226 e-009$ \\
\hline \multirow{2}{*}{$f_{10}$} & \multirow{2}{*}{4} & Mean & $6.27386 e-009$ & $6.25378 e-009$ & $6.40042 e-009$ & $5.92566 \mathrm{e}-009$ \\
\hline & & Std. Dev. & $2.57066 e-009$ & $2.69058 e-009$ & $2.27469 e-009$ & $2.58577 e-009$ \\
\hline \multirow{2}{*}{$f_{11}$} & \multirow{2}{*}{2} & Mean & $4.72646 e-009$ & $4.63412 e-009$ & $4.47891 e-009$ & $3.90594 \mathrm{e}-009$ \\
\hline & & Std. Dev. & $3.03952 e-009$ & $2.74639 e-009$ & $3.01052 e-009$ & $2.94237 e-009$ \\
\hline \multirow{2}{*}{$f_{12}$} & 2 & Mean & $4.65101 e-008$ & $4.65101 e-008$ & $4.65101 e-008$ & $4.65101 e-008$ \\
\hline & 2 & Std. Dev. & 0 & 0 & 0 & 0 \\
\hline$f_{1}$ & 30 & Mean & 0 & 0 & 0 & 0 \\
\hline$J_{13}$ & & Std. Dev. & 0 & 0 & 0 & 0 \\
\hline$f$ & 2 & Mean & $5.04676 e-009$ & $4.58853 e-009$ & $4.67473 e-009$ & $4.51194 e-009$ \\
\hline$J_{14}$ & 2 & Std. Dev. & $2.87757 e-009$ & $3.1021 e-009$ & $2.83989 e-009$ & $2.96938 e-009$ \\
\hline$f_{1}$ & 4 & Mean & 0.00967981 & 0.0121192 & 0.0154611 & 0.0047311 \\
\hline$J_{15}$ & & Std. Dev. & 0.0461986 & 0.048523 & 0.0506461 & 0.0142838 \\
\hline$f$ & 10 & Mean & 0.0320855 & 0.0287587 & 0.0247149 & 0.0239915 \\
\hline$J_{16}$ & 10 & Std. Dev. & 0.023079 & 0.0368454 & 0.0268588 & 0.0306438 \\
\hline$f_{1}$ & 30 & Mean & $9.16118 e-009$ & $9.11199 e-009$ & $9.30319 e-009$ & $9.10653 e-009$ \\
\hline$J_{17}$ & 30 & Std. Dev. & $8.92376 e-010$ & $8.15186 e-010$ & $7.58129 e-010$ & $8.8449 e-010$ \\
\hline$f_{1}$ & 30 & Mean & $9.34244 e-009$ & $9.38564 e-009$ & $9.42362 e-009$ & $9.22727 \mathrm{e}-009$ \\
\hline$J_{18}$ & 30 & Std. Dev. & $5.34739 e-010$ & $4.09769 e-010$ & $4.70872 e-010$ & $7.64185 e-010$ \\
\hline$f$ & 30 & Mean & 1.73273 & 2.07072 & 1.40319 & 1.38389 \\
\hline$J_{19}$ & 30 & Std. Dev. & 1.11967 & 2.26245 & 1.01423 & 1.17433 \\
\hline$f_{20}$ & 30 & Mean & 0 & 0 & 0 & 0 \\
\hline$J_{20}$ & 30 & Std. Dev. & 0 & 0 & 0 & 0 \\
\hline$f$ & 30 & Mean & 0.000697643 & 0.000727313 & 0.000740714 & 0.000656965 \\
\hline$J_{21}$ & 50 & Std. Dev. & 0.00020979 & 0.000291491 & 0.000261093 & 0.0001786 \\
\hline$f$ & 4 & Mean & 0.00405371 & 0.00405371 & 0.00405371 & 0.00405371 \\
\hline$J_{22}$ & 4 & Std. Dev. & $8.89894 e-019$ & $8.44228 e-019$ & $8.89894 e-019$ & $8.44228 e-019$ \\
\hline
\end{tabular}


TABLE 3: Comparison among the $4 \mathrm{DE}$ algorithms on test problems $f_{23}-f_{34}$, where "Mean" indicates the mean function error value and "Std. Dev." stands for the standard deviation. The best results among the four algorithms are shown in boldface.

\begin{tabular}{|c|c|c|c|c|c|c|}
\hline & $D$ & & $\mathrm{DE}_{\mathrm{r}}$ & $\mathrm{DE}_{\mathrm{o}}$ & $\mathrm{DE}_{\mathrm{go}}$ & $\mathrm{DE}_{\mathrm{ar}}$ \\
\hline \multirow{2}{*}{$f_{23}$} & \multirow{2}{*}{4} & Mean & 10.1876 & 10.1876 & 10.1876 & 10.1876 \\
\hline & & Std. Dev. & $5.46751 e-015$ & $5.46751 e-015$ & $5.31347 e-015$ & $5.31347 e-015$ \\
\hline \multirow{2}{*}{$f_{24}$} & \multirow{2}{*}{4} & Mean & 10.4503 & 10.4503 & 10.4503 & 10.4503 \\
\hline & & Std. Dev. & $1.8225 e-015$ & $1.8225 e-015$ & $1.8225 e-015$ & $1.8225 e-015$ \\
\hline \multirow{2}{*}{$f_{25}$} & \multirow{2}{*}{4} & Mean & 10.6103 & 10.6103 & 10.6103 & 10.6103 \\
\hline & & Std. Dev. & $1.8225 e-015$ & $1.8225 e-015$ & $1.8225 e-015$ & $1.8225 e-015$ \\
\hline \multirow{2}{*}{$f_{26}$} & \multirow{2}{*}{2} & Mean & 0.03 & 0.04 & 0.02 & 0.02 \\
\hline & & Std. Dev & 0.171447 & 0.196946 & 0.140705 & 0.140705 \\
\hline \multirow{2}{*}{$f_{27}$} & \multirow{2}{*}{2} & Mean & $2.72741 e-009$ & $2.94925 e-009$ & $2.78605 e-009$ & $2.63247 \mathrm{e}-009$ \\
\hline & & Std. Dev. & $2.76876 e-009$ & $2.8458 e-009$ & $2.79248 e-009$ & $2.76724 e-009$ \\
\hline \multirow{2}{*}{$f_{28}$} & \multirow{2}{*}{30} & Mean & $9.28689 e-009$ & $9.28566 e-009$ & $9.28304 e-009$ & $9.27877 e-009$ \\
\hline & & Std. Dev. & $6.31616 e-010$ & $5.9591 e-010$ & $6.40915 e-010$ & $6.07695 e-010$ \\
\hline \multirow{2}{*}{$f_{29}$} & \multirow{2}{*}{2} & Mean & $4.63641 e-009$ & $4.15234 e-009$ & $4.89748 e-009$ & $3.94368 \mathrm{e}-009$ \\
\hline & & Std. Dev. & $3.24716 e-009$ & $3.01035 e-009$ & $2.81979 e-009$ & $2.7603 e-009$ \\
\hline \multirow{2}{*}{$f_{30}$} & \multirow{2}{*}{5} & Mean & 0.0452575 & 0.0437805 & 0.043716 & 0.0430663 \\
\hline & & Std. Dev. & 0.0220521 & 0.0262655 & 0.0233605 & 0.0220867 \\
\hline \multirow{2}{*}{$f_{31}$} & \multirow{2}{*}{5} & Mean & 0.00523817 & $6.93483 e-009$ & $7.14794 e-009$ & $6.31104 \mathrm{e}-009$ \\
\hline & & Std. Dev. & 0.0523817 & $2.25948 e-009$ & $2.13661 e-009$ & $2.19293 e-009$ \\
\hline \multirow{2}{*}{$f_{32}$} & \multirow{2}{*}{2} & Mean & 0 & 0 & 0 & 0 \\
\hline & & Std. Dev. & 0 & 0 & 0 & 0 \\
\hline \multirow{2}{*}{$f_{33}$} & \multirow{2}{*}{2} & Mean & $4.70104 e-009$ & $4.89609 e-009$ & $4.7038 e-009$ & $4.44655 e-009$ \\
\hline & & Std. Dev. & $2.89836 e-009$ & $2.82738 e-009$ & $3.08112 e-009$ & $2.68808 e-009$ \\
\hline \multirow{2}{*}{$f_{34}$} & \multirow{2}{*}{2} & Mean & $4.75055 e-009$ & $4.73653 e-009$ & $4.52236 e-009$ & $4.23427 \mathrm{e}-009$ \\
\hline & & Std. Dev. & $2.96249 e-009$ & $3.10385 e-009$ & $3.18058 e-009$ & $2.89249 e-009$ \\
\hline
\end{tabular}

TABLE 4: Average ranking of the $4 \mathrm{DE}$ algorithms.

\begin{tabular}{lc}
\hline Algorithm & Ranking \\
\hline $\mathrm{DE}_{\mathrm{ar}}$ & 3.5588235294117663 \\
$\mathrm{DE}_{\mathrm{r}}$ & 2.205882352941176 \\
$\mathrm{DE}_{\mathrm{go}}$ & 2.1470588235294117 \\
$\mathrm{DE}_{\mathrm{o}}$ & 2.0882352941176463 \\
\hline
\end{tabular}

significantly different. In this paper, we only discuss whether the hypotheses is rejected at the 0.05 level of significance. As we can see, all the four tests reject hypotheses 1-3.

Besides the above four tests, we also conduct Wilcoxon's test to recognize significant differences between the behavior of two algorithms $[7,15]$. Table 6 shows the $P$ values of applying Wilcoxon's test among $\mathrm{DE}_{\mathrm{ar}}$ and the other three $\mathrm{DE}$ algorithms. The $P$ values below 0.05 (the significant level) are shown in boldface. From the results, it can be seen that $\mathrm{DE}_{\mathrm{ar}}$ is significantly better than $\mathrm{DE}_{\mathrm{r}}, \mathrm{DE}_{\mathrm{o}}$, and $\mathrm{DE}_{\mathrm{go}}$.

5.3.3. Scalability Test: Effect of Problem Dimensionality. The performance of most EAs (including DE) deteriorates quickly with the growth of the dimensionality of the problem. The main reason is that, in general, the complexity of the problem (search space) increases exponentially with its dimension. Here, we show a scalable test of $\mathrm{DE}_{\mathrm{ar}}$ for $D / 2, D$, and $2 D$ for each scalable function in our test set. Table 7 summarizes the comparison results of the four $\mathrm{DE}$ algorithms for $D / 2$, $D$, and $2 D$. From the results, it can be seen that $D_{a r}$ is not always affected by the growth of dimensionality. For $f_{1}$, $f_{2}, f_{3}, f_{7}, f_{8}, f_{17}, f_{18}, f_{21}$, and $f_{28}, \mathrm{DE}_{\mathrm{ar}}$ achieves similar performance when the dimension increases from $D / 2$ to $2 D$. The performance of $\mathrm{DE}_{\mathrm{ar}}$ deteriorates quickly with the growth of dimension for five functions $\left(f_{4}, f_{5}, f_{6}, f_{19}\right.$, and $\left.f_{20}\right)$. For the remaining one function $\left(f_{13}\right)$, the growth of dimension does not affect the performance of $\mathrm{DE}_{\mathrm{ar}}$.

5.3.4. Effect of Different $k$ Settings. In $\mathrm{DE}_{\mathrm{ar}}$, a new control parameter $k$ (the number of trial individuals) is added to DE's parameters (ps, $F$, and CR). Since $k$ denotes the number of trial individuals, $k$ should be a positive integer. And when $k=1, \mathrm{DE}_{\mathrm{ar}}$ is equal to $\mathrm{DE}_{\mathrm{r}}$. So in our study we restrict $k$ to the positive integers within the range of $[2, \mathrm{ps}]$. As mentioned above, $k$ was fixed to 3 in all experiments. Such a value was set without any effort to find an optimal value. However the performance of $\mathrm{DE}_{\mathrm{ar}}$ may be influenced by different settings of $k$ values.

We investigate the correlation between $k$ and the quality of the final solutions using the Spearman correlation [16]. We repeat the conducted experiments in Section 5.3.3 for $k \epsilon$ $[10,100]$ (since ps $=100$ ) with step size of 10 (i.e., 1,000 trials per function per $k \in\{10,20,30,40,50,60,70,80,90,100\})$. 
TABle 5: Adjusted $P$ values.

\begin{tabular}{|c|c|c|c|c|}
\hline$i$ & Hypothesis & Unadjusted $P$ & $P_{\text {Neme }}$ & $P_{\text {Holm }}$ \\
\hline 1 & $\mathrm{DE}_{\mathrm{o}}$ versus $\mathrm{DE}_{\mathrm{ar}}$ & $2.6442139326214958 e-6$ & $1.5865283595728974 e-5$ & $1.5865283595728974 e-5$ \\
\hline 2 & $\mathrm{DE}_{\mathrm{go}}$ versus $\mathrm{DE}_{\mathrm{ar}}$ & $6.519075482494892 e-6$ & $3.9114452894969356 e-5$ & $3.2595377412474464 e-5$ \\
\hline 3 & $\mathrm{DE}_{\mathrm{r}}$ versus $\mathrm{DE}_{\mathrm{ar}}$ & $1.5536056185614817 e-5$ & $9.32163371136889 e-5$ & $6.214422474245927 e-5$ \\
\hline 4 & $\mathrm{DE}_{\mathrm{r}}$ versus $\mathrm{DE}_{\mathrm{o}}$ & 0.7071142312899601 & 4.24268538773976 & 2.12134269386988 \\
\hline 5 & $\mathrm{DE}_{\mathrm{o}}$ versus $\mathrm{DE}_{\mathrm{go}}$ & 0.8509806870320539 & 5.1058841221923235 & 2.12134269386988 \\
\hline 6 & $\mathrm{DE}_{\mathrm{r}}$ versus $\mathrm{DE}_{\mathrm{go}}$ & 0.8509806870320561 & 5.105884122192337 & 2.12134269386988 \\
\hline$i$ & Hypothesis & $P_{\text {Shaf }}$ & $P_{\text {Berg }}$ & \\
\hline 1 & $\mathrm{DE}_{\mathrm{o}}$ versus $\mathrm{DE}_{\mathrm{ar}}$ & $1.5865283595728974 e-5$ & $1.5865283595728974 e-5$ & \\
\hline 2 & $\mathrm{DE}_{\mathrm{go}}$ versus $\mathrm{DE}_{\mathrm{ar}}$ & $1.9557226447484678 e-5$ & $1.9557226447484678 e-5$ & \\
\hline 3 & $\mathrm{DE}_{\mathrm{r}}$ versus $\mathrm{DE}_{\mathrm{ar}}$ & $4.660816855684445 e-5$ & $3.1072112371229634 e-5$ & \\
\hline 4 & $\mathrm{DE}_{\mathrm{r}}$ versus $\mathrm{DE}_{\mathrm{o}}$ & 2.12134269386988 & 2.12134269386988 & \\
\hline 5 & $\mathrm{DE}_{\mathrm{o}}$ versus $\mathrm{DE}_{\mathrm{go}}$ & 2.12134269386988 & 2.12134269386988 & \\
\hline 6 & $\mathrm{DE}_{\mathrm{r}}$ versus $\mathrm{DE}_{\mathrm{go}}$ & 2.12134269386988 & 2.12134269386988 & \\
\hline
\end{tabular}

TABLE 6: Wilcoxon test between $\mathrm{DE}_{\mathrm{ar}}$ and the other three $\mathrm{DE}$ algorithms on functions $f_{1}-f_{34}$. The $P$ values below 0.05 are shown in boldface.

\begin{tabular}{lc}
\hline $\mathrm{DE}_{\mathrm{ar}}$ versus & $P$ values \\
\hline $\mathrm{DE}_{\mathrm{r}}$ & $\mathbf{0 . 0 0 0 0 1 2}$ \\
$\mathrm{DE}_{\mathrm{go}}$ & $\mathbf{0 . 0 0 0 0 1 8}$ \\
$\mathrm{DE}_{\mathrm{o}}$ & $\mathbf{0 . 0 0 0 0 1 2}$ \\
\hline
\end{tabular}

For the limitation of space, we do not show all the results of the final solutions; only the final solutions obtained on $f_{2}$ and $f_{3}$ are shown in Table 8 for illustration. But almost a similar behavior has been observed for all functions that the quality of the final solution is better than that of $\mathrm{DE}_{\mathrm{r}}, \mathrm{DE}_{\mathrm{o}}$, and $\mathrm{DE}_{\mathrm{go}}$ when $k>1$.

Table 9 shows the Spearman correlation test results between $k$ and the final solutions obtained on each test function. As seen, there is not a significant correlation between $k$ and the quality of the final solutions. It means that $k$, like other control parameters of DE, has a problemoriented value. Since the larger the $k$ value is, the more time to initialize the population is required, especially when the dimensionality of the problem is also large. Our limited experiments suggest to use a small value of $k$.

\section{Conclusions}

This paper employs the concept of adaptive randomness (AR) for population initialization. The main idea of $A R$ is to make use of the difference between individuals to make them more evenly spread over the search space and then a better approximation for the current candidate solution is obtained. In order to investigate the performance of the AR-based population initialization, the classical DE has been utilized. By embedding AR within $\mathrm{DE}, \mathrm{DE}_{\mathrm{ar}}$ was proposed. Experiments are conducted on 34 benchmark functions. The experimental results can be summarized as follows.

(i) $\mathrm{DE}_{\mathrm{ar}}$ is compared with other DE algorithms such as $\mathrm{DE}$ with random initialization $\left(\mathrm{DE}_{\mathrm{r}}\right), \mathrm{DE}$ with opposition-based learning $\left(\mathrm{DE}_{\mathrm{o}}\right)$, and $\mathrm{DE}$ with generalized opposition-based learning $\left(\mathrm{DE}_{\mathrm{go}}\right)$ with respect to the convergence speed and robustness. The results demonstrate that $\mathrm{DE}_{\mathrm{ar}}$ performs better than the other three DE algorithms at least on $64.7 \%$ of the test functions. Although the other three DE algorithms outperform $\mathrm{DE}_{\mathrm{ar}}$ on some functions, their success rates are always worse.

(ii) $\mathrm{DE}_{\mathrm{ar}}$ is further compared with $\mathrm{DE}_{\mathrm{r}}, \mathrm{DE}_{\mathrm{o}}$, and $\mathrm{DE}_{\mathrm{go}}$ with respect to the quality of the final solutions. The results show that $\mathrm{DE}_{\mathrm{ar}}$ performs better than the other three DE algorithms on the majority (about 70.5\%) of test functions. And on the rest of functions, $\mathrm{DE}_{\mathrm{ar}}$ obtains no worse results than the results of the other three DE algorithms. Statistical comparisons also show that $\mathrm{DE}_{\mathrm{ar}}$ is the best of the four DE algorithms.

(iii) A scalability test of $\mathrm{DE}_{\mathrm{ar}}$ over 15 test functions with different problem dimensions $(D / 2, D$, and $2 D)$ are conducted. The 15 functions are scalable and chosen from the 34 test functions. The results show that $\mathrm{DE}_{\mathrm{ar}}$ is not always affected by the growth of dimensionality. For 9 functions, $\mathrm{DE}_{\mathrm{ar}}$ achieves similar performance when the dimension increases from $D / 2$ to $2 D$. For 5 functions, the performance of $\mathrm{DE}_{\mathrm{ar}}$ deteriorates quickly with the growth of dimension while for the remaining 1 function, the growth of dimension does not affect the performance of $\mathrm{DE}_{\mathrm{ar}}$.

(iv) The influence of $k$ (number of trial individuals) was studied by investigating the Spearman correlation between $k$ and the quality of the final solutions. The results obtained on the 34 test functions show that there is not a significant correlation between $k$ and the quality of the final solutions. But the quality of the final solution is better than that of the other three DE algorithms when $k>1$.

The main motivation of the current work was the introduction of the concept of adaptive randomness for population initialization. Although this paper only embeds the AR within 
TABLE 7: Mean function error values of $\mathrm{DE}_{\mathrm{ar}}$ for $D / 2, D$, and $2 D$ for each scalable function in our test set.

\begin{tabular}{|c|c|c|c|c|c|c|c|}
\hline \multirow{2}{*}{$F$} & \multicolumn{3}{|c|}{$\mathrm{DE}_{\mathrm{ar}}$} & \multirow{2}{*}{$F$} & \multicolumn{3}{|c|}{$\mathrm{DE}_{\mathrm{ar}}$} \\
\hline & $D / 2$ & $D$ & $2 D$ & & $D / 2$ & $D$ & $2 D$ \\
\hline$f_{1}$ & $8.22347 e-009$ & $8.77417 e-009$ & $9.09346 e-009$ & $f_{13}$ & 0 & 0 & 0 \\
\hline$f_{2}$ & $8.27444 e-009$ & $8.92437 e-009$ & $9.25353 e-009$ & $f_{17}$ & $8.20248 e-009$ & $9.10653 e-009$ & $7.33223 e-006$ \\
\hline$f_{3}$ & $8.33435 e-009$ & $9.33293 e-009$ & $9.3625 e-009$ & $f_{18}$ & $9.14822 e-009$ & $9.22727 e-009$ & $9.65751 e-009$ \\
\hline$f_{4}$ & $8.30962 e-009$ & $9.11962 e-009$ & 1.50496 & $f_{19}$ & $9.05241 e-009$ & 1.38389 & 11.5557 \\
\hline$f_{5}$ & $7.0724 e-009$ & 0.049748 & 2.53715 & $f_{20}$ & 0 & 0 & 0.35 \\
\hline$f_{6}$ & $8.04869 e-009$ & $8.80458 e-009$ & 0.000369811 & $f_{21}$ & 0.000222243 & 0.000656965 & 0.00434487 \\
\hline$f_{7}$ & $5.81354 e-009$ & $6.20522 e-009$ & $6.63678 e-009$ & $f_{28}$ & $8.92662 e-009$ & $9.27877 e-009$ & $9.54968 e-009$ \\
\hline$f_{8}$ & $9.00816 e-009$ & $9.25572 e-009$ & $9.64823 e-009$ & & & & \\
\hline
\end{tabular}

Note. The values of $D$ for each test function are shown as those in Table 1.

TABLE 8: Final solutions obtained on $f_{2}$ and $f_{3}$ on interval $[10,100]$ of $k$ with step size of 10 .

\begin{tabular}{cccccr}
\hline & $k=10$ & $k=20$ & $k=30$ & $k=40$ & $k=50$ \\
\hline$f_{2}$ & $8.78572 e-009$ & $8.85414 e-009$ & $8.77919 e-009$ & $8.7689 e-009$ & $8.79035 e-009$ \\
$f_{3}$ & $8.67476 e-009$ & $8.8475 e-009$ & $8.83617 e-009$ & $8.85011 e-009$ & $8.79553 e-009$ \\
\hline & $k=60$ & $k=70$ & $k=80$ & $k=90$ & $k=100$ \\
\hline$f_{2}$ & $8.83882 e-009$ & $8.64561 e-009$ & $8.75898 e-009$ & $8.73418 e-009$ & $8.8088 e-009$ \\
$f_{3}$ & $8.74038 e-009$ & $8.8981 e-009$ & $8.81404 e-009$ & $8.76988 e-009$ & $8.80807 e-009$ \\
\hline
\end{tabular}

classical DE, the idea is general enough to be applied to all other population-based methods (e.g., GA and PSO). Further since AR is new, studies are still required to investigate its benefits, weaknesses, and limitations. The current work can be considered as a first step in applying AR.

\section{Appendix}

\section{List of Benchmark Functions}

The 34 test functions we employed are given below. All the functions used in this paper are to be minimized.

(1) Sphere Model. Consider

$$
f_{1}(X)=\sum_{i=1}^{n} x_{i}^{2}
$$

where $x_{i} \in[-5.12,5.12]$ and the global optimum is 0 at $(0$, $0, \ldots, 0) . f_{1}$ is a unimodal, scalable, convex, and easy function.

(2) Axis Parallel Hyperellipsoid. Consider

$$
f_{2}(X)=\sum_{i=1}^{n} i x_{i}^{2}
$$

where $x_{i} \in[-5.12,5.12]$ and the global optimum is 0 at $(0$, $0, \ldots, 0) . f_{2}$ is a unimodal, scalable, convex, and easy function.

(3) Schwefel's Problem 1.2. Consider

$$
f_{3}(X)=\sum_{i=1}^{n}\left(\sum_{j=1}^{i} x_{j}\right)^{2}
$$

where $x_{i} \in[-65,65]$ and the global optimum is 0 at $(0,0, \ldots, 0) . f_{3}$ is a unimodal and scalable function.

(4) Rosenbrock's Valley. Consider

$$
f_{4}(X)=\sum_{i=1}^{n-1}\left[100\left(x_{i+1}-x_{i}^{2}\right)^{2}+\left(1-x_{i}\right)^{2}\right],
$$

where $x_{i} \in[-2,2]$ and the global optimum is 0 at $(1,1, \ldots, 1)$. $f_{4}$ is a nonconvex unimodal function. Its optimum is inside a long, narrow, parabolic shaped flat valley.

(5) Rastrigin's Function. Consider

$$
f_{5}(X)=10 n+\sum_{i=1}^{n}\left(x_{i}^{2}-10 \cos \left(2 \pi x_{i}\right)\right)
$$

where $x_{i} \in[-5.12,5.12]$ and the global optimum is 0 at $(0,0, \ldots, 0) . f_{5}$ is highly mutimodel.

(6) Griewangk's Function. Consider

$$
f_{6}(X)=\sum_{i=1}^{n} \frac{x_{i}^{2}}{4000}-\prod_{i=1}^{n} \cos \left(\frac{x_{i}}{\sqrt{i}}\right)+1
$$

where $x_{i} \in[-600,600]$ and the global optimum is 0 at $(0,0, \ldots, 0)$.

(7) Sum of Different Powers. Consider

$$
f_{7}(X)=\sum_{i=1}^{n}\left|x_{i}\right|^{(i+1)}
$$


TABLE 9: Spearman's correlation test results between $k$ and the final solutions on all the test functions.

\begin{tabular}{cccccccccccc}
\hline & SC & & SC & & SC & & SC & & SC & SC \\
\hline$f_{1}$ & -0.399 & $f_{7}$ & 0.394 & $f_{13}$ & 0.068 & $f_{19}$ & -0.261 & $f_{25}$ & -0.327 & $f_{31}$ & -0.304 \\
$f_{2}$ & -0.333 & $f_{8}$ & 0.576 & $f_{14}$ & -0.124 & $f_{20}$ & 0.287 & $f_{26}$ & -0.108 & $f_{32}$ & 0.196 \\
$f_{3}$ & -0.018 & $f_{9}$ & -0.321 & $f_{15}$ & -0.120 & $f_{21}$ & 0.168 & $f_{27}$ & -0.282 & $f_{33}$ & 0.292 \\
$f_{4}$ & 0.624 & $f_{10}$ & -0.394 & $f_{16}$ & 0.317 & $f_{22}$ & 0.127 & $f_{28}$ & -0.267 & $f_{34}$ & -0.174 \\
$f_{5}$ & -0.065 & $f_{11}$ & -0.333 & $f_{17}$ & 0.258 & $f_{23}$ & -0.157 & $f_{29}$ & 0.260 & \\
$f_{6}$ & 0.060 & $f_{14}$ & -0.245 & $f_{18}$ & 0.197 & $f_{24}$ & -0.388 & $f_{30}$ & -0.142 & \\
\hline
\end{tabular}

Note. SC denotes the correlation coefficient of the Spearman correlation test.

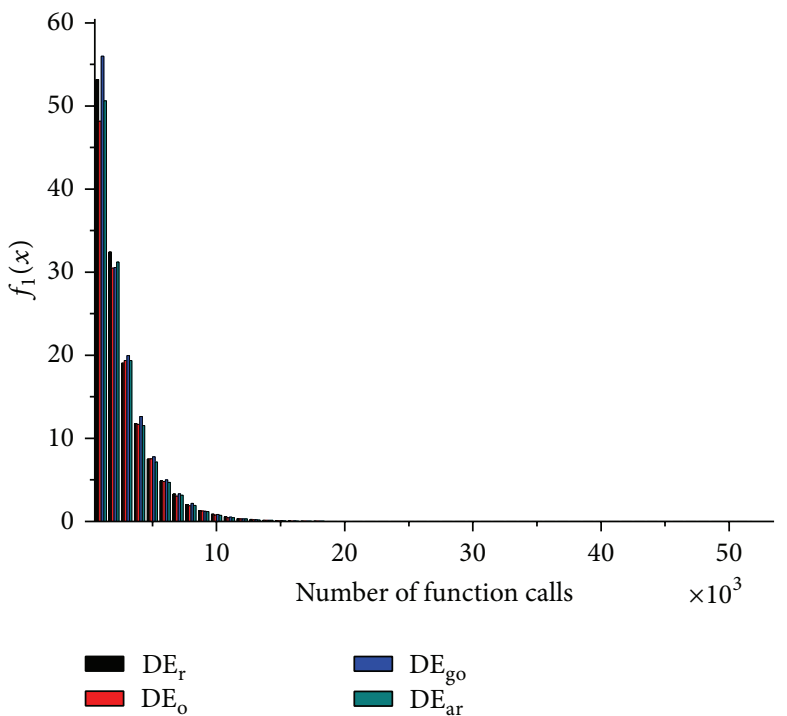

(a) $f_{1}$

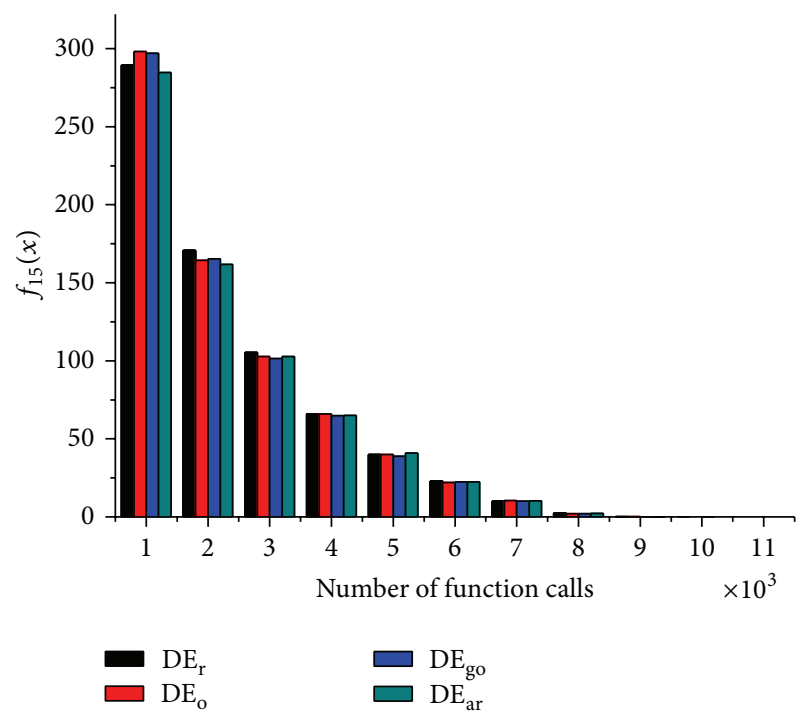

(c) $f_{13}$

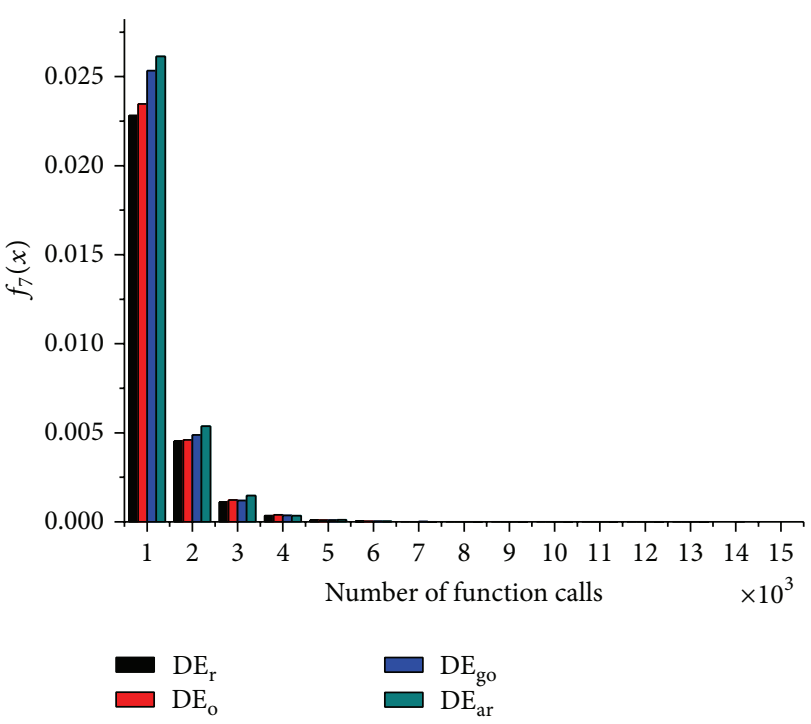

(b) $f_{7}$

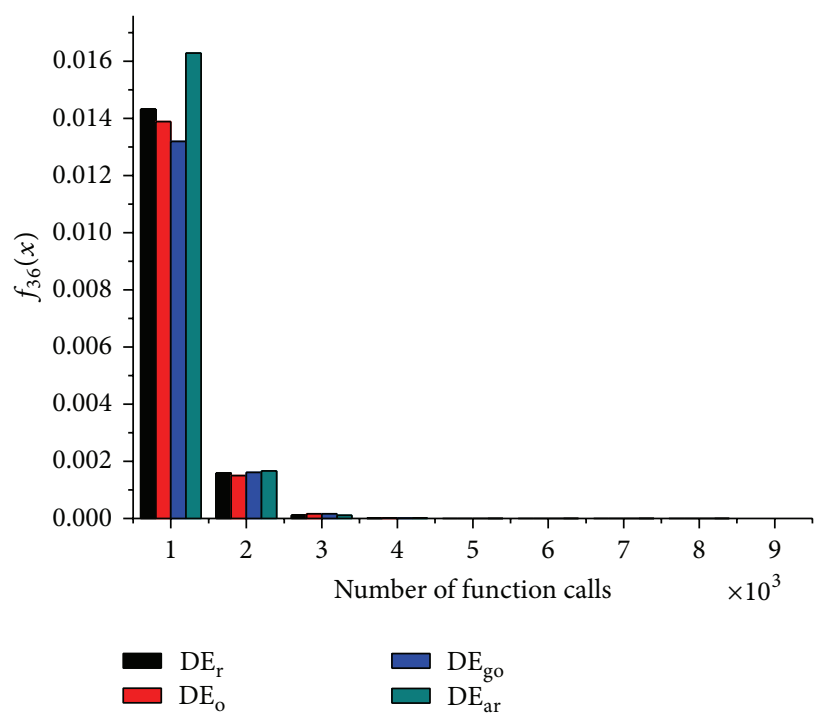

(d) $f_{33}$

FIGURE 3: Some sample bar charts for the performance comparison of the $4 \mathrm{DE}$ algorithms. The values are calculated at every 1,000 function calls. 
where $x_{i} \in[-1,1]$ and the global optimum is 0 at $(0,0, \ldots, 0)$. $f_{7}$ is a unimodal and scalable function.

(8) Ackley's Problem. Consider

$$
\begin{aligned}
f_{8}(X)= & -20 \exp \left(-0.2 \sqrt{\frac{\sum_{i=1}^{n} x_{i}^{2}}{n}}\right) \\
& -\exp \left(\frac{\sum_{i=1}^{n} \cos \left(2 \pi x_{i}\right)}{n}\right)+20+e,
\end{aligned}
$$

where $x_{i} \in[-32,32]$ and the global optimum is 0 at $(0,0$, $\ldots, 0)$.

(9) Beale’s Function. Consider

$$
\begin{aligned}
f_{9}(X)= & {\left[1.5-x_{1}\left(1-x_{2}\right)\right]^{2}+\left[2.25-x_{1}\left(1-x_{2}^{2}\right)\right]^{2} } \\
& +\left[2.625-x_{1}\left(1-x_{2}^{3}\right)\right]^{2},
\end{aligned}
$$

where $x_{i} \in[-4.5,4.5]$ and the global optimum is 0 at $(3,0.5)$.

(10) Colville's Function. Consider

$$
\begin{aligned}
f_{10}(X)= & 100\left(x_{2}-x_{1}^{2}\right)^{2}+\left(1-x_{1}\right)^{2}+90\left(x_{4}-x_{3}^{2}\right)^{2} \\
& +\left(1-x_{3}\right)^{2}+10.1\left(\left(x_{2}-1\right)^{2}+\left(x_{4}-1\right)^{2}\right) \\
& +19.8\left(x_{2}-1\right)\left(x_{4}-1\right),
\end{aligned}
$$

where $x_{i} \in[-10,10]$ and the global optimum is 0 at $(1,1,1,1)$.

\section{(11) Easom's Function. Consider}

$$
f_{11}(X)=-\cos \left(x_{1}\right) \cos \left(x_{2}\right) \exp \left(-\left(x_{1}-\pi\right)^{2}-\left(x_{2}-\pi\right)^{2}\right) \text {, }
$$

where $x_{i} \in[-100,100]$ and the global optimum is 0 at $(\pi, \pi)$. $f_{11}$ is unimodal and its global minimum lays in a narrow area relative to the search space.

\section{(12) Six-Hump Camel Back Function. Consider}

$$
\begin{aligned}
f_{12}(X)= & 4 x_{1}^{2}-2.1 x_{1}^{4}+\frac{1}{3} x_{1}^{6}+x_{1} x_{2}-4 x_{2}^{2} \\
& +4 x_{2}^{4}+1.0316285,
\end{aligned}
$$

where $x_{i} \in[-5,5]$ and the global optimum is 0 at $(0.0898,-0.7126)$ and $(-0.0898,0.7126) . f_{12}$ has six local minima, two of them are global.

(13) Levy's Function. Consider

$$
\begin{aligned}
f_{13}(X)= & \sin ^{2}\left(3 \pi x_{1}\right)+\sum_{i=1}^{n-1}\left(x_{i}-1\right)^{2}\left(1+\sin ^{2}\left(3 \pi x_{i+1}\right)\right) \\
& +\left(x_{n}-1\right)\left(1+\sin ^{2}\left(2 \pi x_{n}\right)\right),
\end{aligned}
$$

where $x_{i} \in[-10,10]$ and the global optimum is 0 at $(1,1$, $\ldots, 1) . f_{13}$ has about $15^{n}$ local minima.

(14) Matyas Function. Consider

$$
f_{14}(X)=0.26\left(x_{1}^{2}+x_{2}^{2}\right)-0.48 x_{1} x_{2},
$$

where $x_{i} \in[-10,10]$ and the global optimum is 0 at $(0,0) . f_{14}$ is unimodal.

(15) Perm Function. Consider

$$
f_{15}(X)=\sum_{k=1}^{n}\left[\sum_{i=1}^{n}\left(i^{k}+0.5\right)\left(\left(\frac{1}{i} x_{i}\right)^{k}-1\right)\right]^{2},
$$

where $x_{i} \in[-n, n]$ and the global optimum is 0 at $(1,2,3$, $\ldots, n) . f_{15}$ is unimodal.

(16) Michalewicz's Function. Consider

$$
f_{16}(X)=-\sum_{i=1}^{n} \sin \left(x_{i}\right)\left(\sin \left(\frac{i x_{i}^{2}}{\pi}\right)\right)^{2 m}+9.66015
$$

where $x_{i} \in[0, \pi], m=10, n=10$, and the global optimum is 0 .

(17) Zakharov's Function. Consider

$$
f_{17}(X)=\sum_{i=1}^{n} x_{i}^{2}+\left(\sum_{i=1}^{n} 0.5 i x_{i}\right)^{2}+\left(\sum_{i=1}^{n} 0.5 i x_{i}\right)^{4},
$$

where $x_{i} \in[-5,10]$ and the global optimum is 0 at $(0,0, \ldots, 0) . f_{17}$ is unimodal.

(18) Schwefel's Problem 2.22. Consider

$$
f_{18}(X)=\sum_{i=1}^{n}\left|x_{i}\right|+\prod_{i=1}^{n}\left|x_{i}\right|,
$$

where $x_{i} \in[-10,10]$ and the global optimum is 0 at $(0,0$, $\ldots, 0) . f_{18}$ is unimodal.

(19) Schwefel's Problem 2.21. Consider

$$
f_{19}(X)=\max \left\{\left|x_{i}\right|, 1 \leq i \leq n\right\},
$$

where $x_{i} \in[-100,100]$ and the global optimum is 0 at $(0,0$, $\ldots, 0) . f_{19}$ is unimodal.

(20) Step Function. Consider

$$
f_{20}(X)=\sum_{i=1}^{n}\left(\left\lfloor x_{i}+0.5\right\rfloor\right)^{2},
$$

where $x_{i} \in[-100,100]$ and the global optimum is 0 when $x_{i} \in[-0.5,0.5]$.

(21) Noisy Quartic Function. Consider

$$
f_{21}(X)=\sum_{i=1}^{n} i x_{i}^{4}+\operatorname{random}[0,1),
$$


where $x_{i} \in[-1.28,1.28]$ and the global optimum is 0 at $(0$, $0, \ldots, 0)$.

\section{(22) Kowalik's Function. Consider}

$$
f_{22}(X)=\sum_{i=1}^{11}\left[a_{i}-\frac{x_{1}\left(b_{i}^{2}+b_{i} x_{2}\right)}{b_{i}^{2}+b_{i} x_{3}+x_{4}}\right]^{2},
$$

where $x_{i} \in[-5,5], \alpha=[0.1957,0.1947,0.1735,0.1600,0.0844$, $0.0627,0.0456,0.0342,0.0323,0.0235,0.0246], b^{-1}=[0.25$, $0.5,1,2,4,6,8,10,12,14,16]$, and the global optimum is 0.0003075 at $(0.19,0.19,0.12,0.14) . f_{22}$ is multimodal.

(23) Shekel 5 Problem. Consider

$$
f_{23}(X)=-\sum_{i=1}^{5} \frac{1}{\sum_{j=1}^{4}\left(x_{j}-a_{i j}\right)^{2}+c_{i}}+10.1499
$$

where

$$
\begin{gathered}
x_{j} \in[0,10], \\
c_{i}=[0.1,0.2,0.2,0.4,0.4], \\
a_{i j}=\left[\begin{array}{llll}
4 & 4 & 4 & 4 \\
1 & 1 & 1 & 1 \\
8 & 8 & 8 & 8 \\
6 & 6 & 6 & 6 \\
3 & 7 & 3 & 7
\end{array}\right],
\end{gathered}
$$

and the global optimum is 0 at $(4,4,4,4)$.

(24) Shekel 7 Problem. Consider

$$
f_{24}(X)=-\sum_{i=1}^{7} \frac{1}{\sum_{j=1}^{4}\left(x_{j}-a_{i j}\right)^{2}+c_{i}}+10.3999
$$

where

$$
\begin{gathered}
x_{i} \in[0,10], \\
c_{i}=[0.1,0.2,0.2,0.4,0.4,0.6,0.3], \\
a_{i j}=\left[\begin{array}{llll}
4 & 4 & 4 & 4 \\
1 & 1 & 1 & 1 \\
8 & 8 & 8 & 8 \\
6 & 6 & 6 & 6 \\
3 & 7 & 3 & 7 \\
2 & 9 & 2 & 9 \\
5 & 5 & 3 & 3
\end{array}\right]
\end{gathered}
$$

and the global optimum is 0 at $(4,4,4,4)$.

(25) Shekel 10 Problem. Consider

$$
f_{25}(X)=-\sum_{i=1}^{10} \frac{1}{\sum_{j=1}^{4}\left(x_{j}-a_{i j}\right)^{2}+c_{i}}+10.5319
$$

where

$$
\begin{gathered}
x_{i} \in[0,10], \\
c_{i}=[0.1,0.2,0.2,0.4,0.4,0.6,0.3,0.7,0.5,0.5], \\
a_{i j}=\left[\begin{array}{cccc}
4 & 4 & 4 & 4 \\
1 & 1 & 1 & 1 \\
8 & 8 & 8 & 8 \\
6 & 6 & 6 & 6 \\
3 & 7 & 3 & 7 \\
2 & 9 & 2 & 9 \\
5 & 5 & 3 & 3 \\
8 & 1 & 8 & 1 \\
6 & 2 & 6 & 2 \\
7 & 3.6 & 7 & 3.6
\end{array}\right]
\end{gathered}
$$

and the global optimum is 0 at $(4,4,4,4)$.

(26) Tripod Function. Consider

$$
\begin{aligned}
f_{26}(X)= & p\left(x_{2}\right)\left(1+p\left(x_{1}\right)\right) \\
& +\left|\left(x_{1}+50 p\left(x_{2}\right)\left(1-2 p\left(x_{1}\right)\right)\right)\right| \\
& +\left|\left(x_{2}+50\left(1-2 p\left(x_{2}\right)\right)\right)\right|,
\end{aligned}
$$

where $x_{i} \in[-100,100], p(x)=1$ for $x \leq 0$; otherwise $p(x)=$ 0 , and the global optimum is 0 at $(0,-50)$.

(27) 4th De Jong. Consider

$$
f_{27}(X)=\sum_{i=1}^{n} i x_{i}^{4}
$$

where $x_{i} \in[-1.28,1.28]$ and the global optimum is 0 at $(0$, $0, \ldots, 0)$.

(28) Alpine Function. Consider

$$
f_{28}(X)=\sum_{i=1}^{n}\left|x_{i} \sin \left(x_{i}\right)+0.1 x_{i}\right|,
$$

where $x_{i} \in[-10,10]$ and the global optimum is 0 at $(0,0$, $\ldots, 0) . f_{28}$ is multimodal and not symmetrical.

(29) Schaffer's Function 6. Consider

$$
f_{29}(X)=0.5+\frac{\sin ^{2} \sqrt{\left(x_{1}^{2}+x_{2}^{2}\right)}-0.5}{1+0.01\left(x_{1}^{2}+x_{2}^{2}\right)^{2}},
$$

where $x_{i} \in[-10,10]$ and the global optimum is 0 at $(0,0) . f_{29}$ is multimodal.

(30) Pathological Function. Consider

$$
f_{30}(X)=\sum_{i=1}^{n-1}\left(0.5+\frac{\sin ^{2} \sqrt{\left(100 x_{i}^{2}+x_{i+1}^{2}\right)}-0.5}{1+0.001\left(x_{i}^{2}-2 x_{i} x_{i+1}+x_{i+1}^{2}\right)^{2}}\right),
$$


where $x_{i} \in[-100,100]$ and the global optimum is 0 at $(0$, $0, \ldots, 0) . f_{30}$ is multimodal and extremely complex.

(31) Inverted Cosine Wave Function. Consider

$$
\begin{aligned}
f_{31}(X)=-\sum_{i=1}^{n-1}( & \exp \left(\frac{-\left(x_{i}^{2}+x_{i+1}^{2}+0.5 x_{i} x_{i+1}\right)}{8}\right) \\
& \left.\times \cos \left(4 \sqrt{x_{i}^{2}+x_{i+1}^{2}+0.5 x_{i} x_{i+1}}\right)\right)+n-1,
\end{aligned}
$$

where $x_{i} \in[-5,5]$ and the global optimum is $1-n$ at $(0,0$, $\ldots, 0) . f_{31}$ is multimodal.

\section{(32) Aluffi-Pentini's Problem. Consider}

$$
f_{32}(X)=0.25 x_{1}^{4}-0.5 x_{1}^{2}+0.1 x_{1}+0.5 x_{2}^{2}+0.3523
$$

where $x_{i} \in[-10,10]$ and the global optimum is 0 at $(-1.0465$, $0)$.

\section{(33) Becker and Lago Problem. Consider}

$$
f_{33}(X)=\left(\left|x_{1}\right|-5\right)^{2}+\left(\left|x_{2}\right|-5\right)^{2},
$$

where $x_{i} \in[-10,10]$ and the global optimum is 0 at $( \pm 5, \pm 5)$.

\section{(34) Bohachevsky 1 Problem. Consider}

$$
f_{34}(X)=x_{1}^{2}+2 x_{2}^{2}-0.3 \cos \left(3 \pi x_{1}\right) 0.4 \cos \left(4 \pi x_{2}\right)+0.3,
$$

where $x_{i} \in[-50,50]$ and the global optimum is 0 at $(0,0)$.

\section{Conflict of Interests}

The authors declare that there is no conflict of interests regarding the publication of this paper.

\section{Acknowledgments}

This work was supported by the National Natural Science Foundation of China (nos. 61202048 and 61202200), the Commonwealth Project of Science and Technology Department of Zhejiang Province (no. 2014C23008), the Zhejiang Provincial Nature Science Foundation of China (no. LY13F020010), and the Open Foundation of State Key Laboratory of Software Engineering of Wuhan University of China (no. SKLSE-2012-09-21).

\section{References}

[1] J. H. Holland, Adaptation in Natural and Artificial Systems: An Introductory Analysis with Applications to Biology, Control, and Artificial Intelligence, MIT Press, Cambridge, UK, 1992.

[2] R. Storn and K. Price, "Differential evolution: a simple and efficient heuristic for global optimization over continuous spaces," Journal of Global Optimization, vol. 11, no. 4, pp. 341-359, 1997.
[3] K. Price, R. Storn, and J. Lampinen, Differential EvolutionA Practical Approach to Global Optimization, Springer, Berlin, Germany, 2005.

[4] H. Maaranen, K. Miettinen, and M. M. Mäkelä, "Quasi-random initial population for genetic algorithms," Computers \& Mathematics with Applications, vol. 47, no. 12, pp. 1885-1895, 2004.

[5] S. Rahnamayan, H. R. Tizhoosh, and M. M. A. Salama, "A novel population initialization method for accelerating evolutionary algorithms," Computers \& Mathematics with Applications, vol. 53, no. 10, pp. 1605-1614, 2007.

[6] H. Wang, Z. Wu, Y. Liu, J. Wang, D. Jiang, and L. Chen, "Space transformation search: a new evolutionary technique," in Proceedings of the 1st ACM/SIGEVO Summit on Genetic and Evolutionary Computation (GEC '09), pp. 537-544, Shanghai, China, June 2009.

[7] H. Wang, Z. Wu, and S. Rahnamayan, "Enhanced oppositionbased differential evolution for solving high-dimensional continuous optimization problems," Soft Computing, vol. 15, no. 11, pp. 2127-2140, 2011.

[8] G. C. Onwubolu and B. V. Babu, New Optimiza tion Techniques in Engineering, Springer, Berlin, Germany, 2004.

[9] J. Andre, P. Siarry, and T. Dognon, "An improvement of the standard genetic algorithm fighting premature convergence in continuous optimization," Advances in Engineering Software, vol. 32, no. 1, pp. 49-60, 2001.

[10] J. Brest, S. Greiner, B. Bošković, M. Mernik, and V. Zumer, "Self-adapting control parameters in differential evolution: a comparative study on numerical benchmark problems," IEEE Transactions on Evolutionary Computation, vol. 10, no. 6, pp. 646-657, 2006.

[11] X. Yao, Y. Liu, and G. Lin, "Evolutionary programming made faster," IEEE Transactions on Evolutionary Computation, vol. 3, no. 2, pp. 82-102, 1999.

[12] C.-Y. Lee and X. Yao, "Evolutionary programming using mutations based on the Levy probability distribution," IEEE Transactions on Evolutionary Computation, vol. 8, no. 1, pp. 1-13, 2004.

[13] R. S. Rahnamayan, H. R. Tizhoosh, and M. M. A. Salama, "Opposition-based differential evolution," IEEE Transactions on Evolutionary Computation, vol. 12, no. 1, pp. 64-79, 2008.

[14] P. N. Suganthan, N. Hansen, J. J. Liang et al., "Problem definitions and evaluation criteria for the CEC 2005 special session on real-parameter optimization," Tech. Rep. 2005005, Nanyang Tech. Univ., Singapore and KanGAL, Kanpur Genetic Algorithms Lab., IIT, Kanpur, India, May 2005.

[15] S. Garcia, A. Fernandez, J. Luengo, and F. Herrera, "Advanced nonparametric tests for multiple comparisons in the design of experiments in computational intelligence and data mining: experimental analysis of power," Information Sciences, vol. 180, no. 10, pp. 2044-2064, 2010.

[16] C. R. Kothari, Research Methodology: Methods and Techniques, New Age International, New Delhi, India, 2007. 


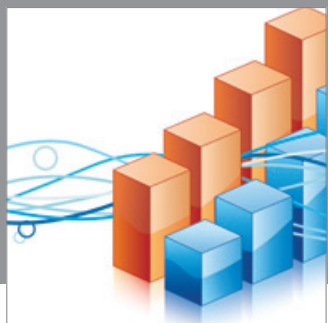

Advances in

Operations Research

mansans

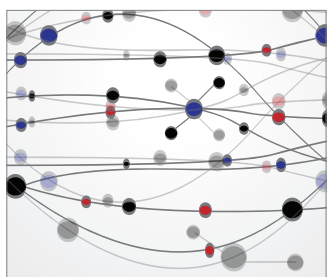

The Scientific World Journal
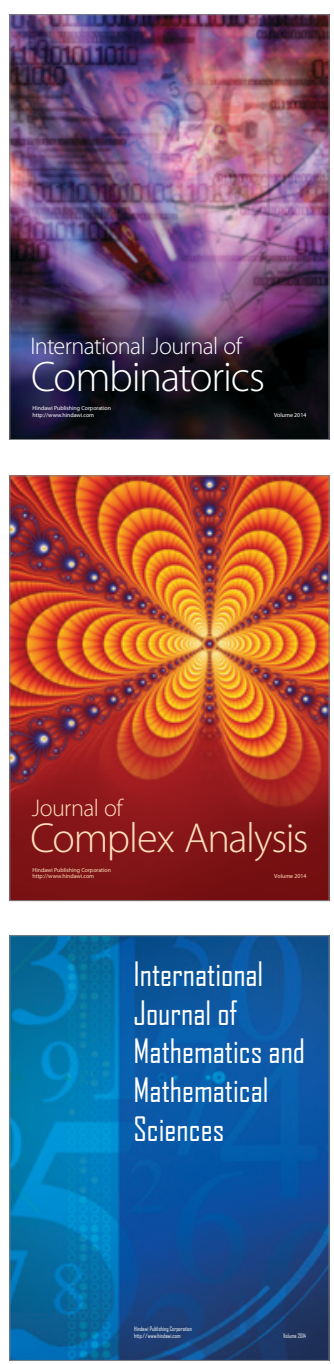
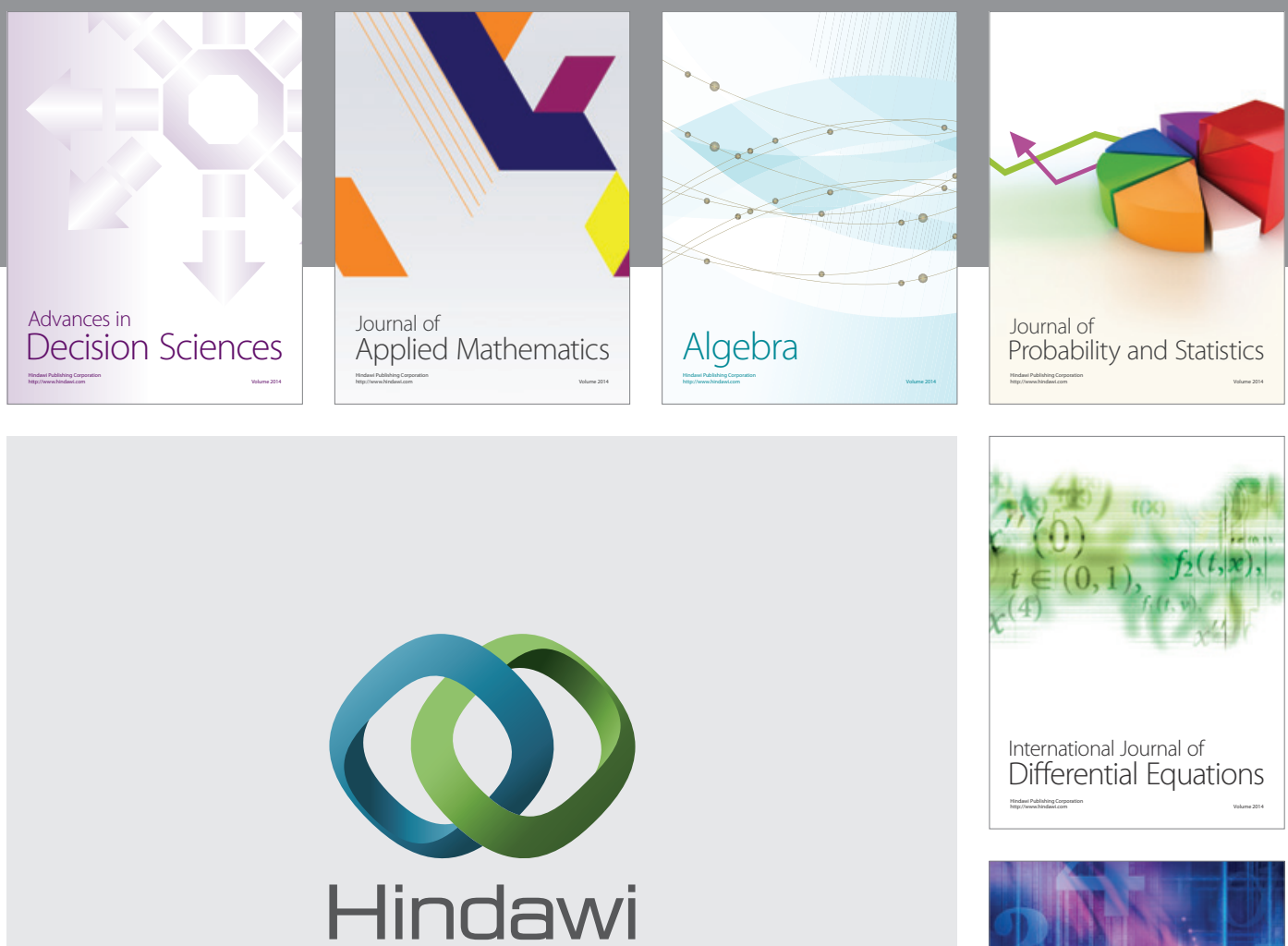

Submit your manuscripts at http://www.hindawi.com
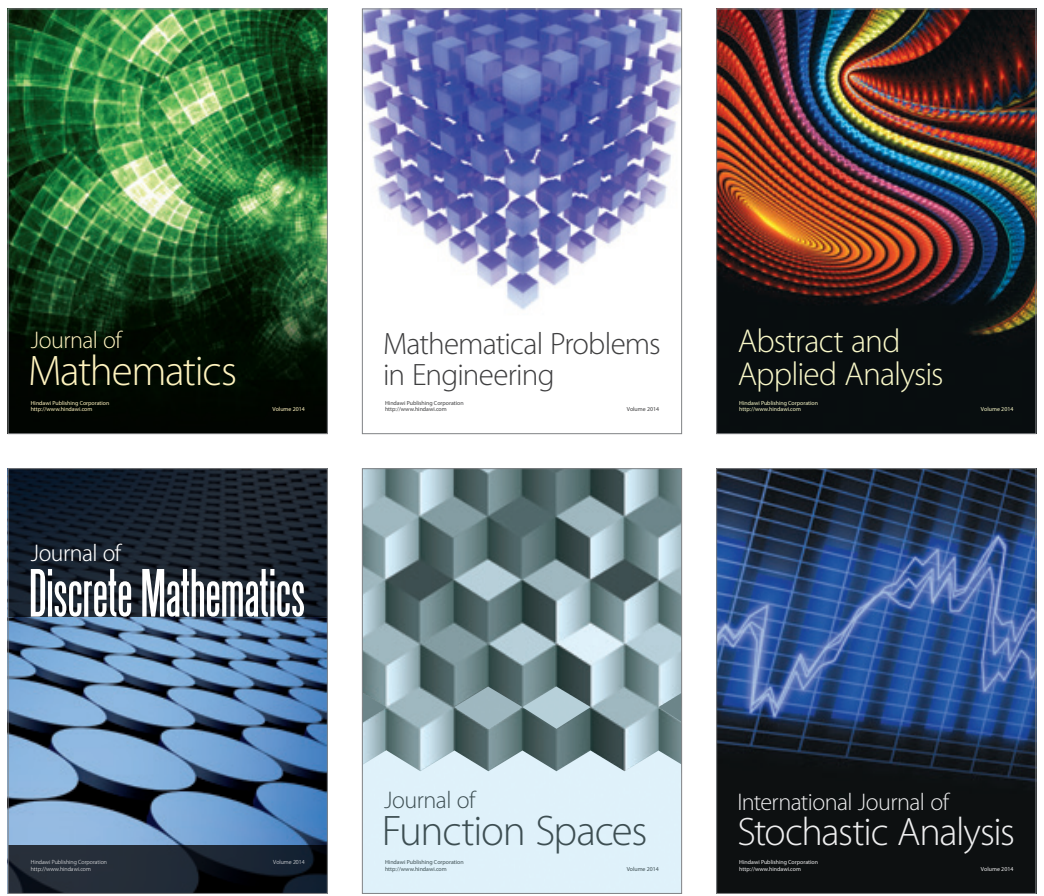

Journal of

Function Spaces

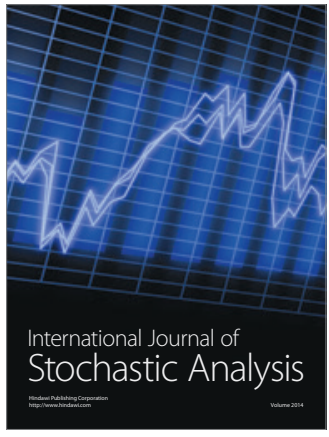

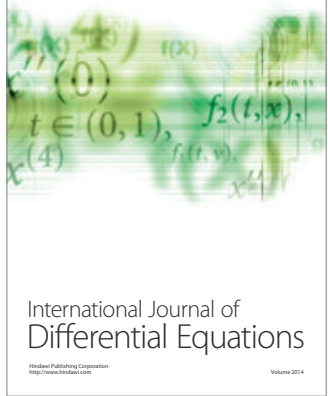
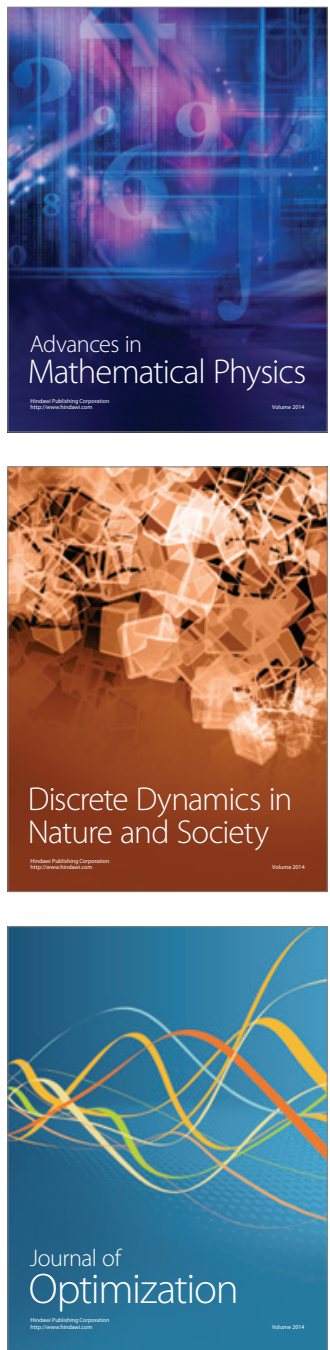